\title{
Characterization of Atomization Processes in Suspension/Emulsion Sprays
}

\author{
Walter Schäfer ${ }^{1}$ and Cameron Tropea ${ }^{2}$ \\ 1. Research \& Development Department, Advanced Optical Measurement Systems GmbH, Darmstadt-Griesheim 64347, Germany \\ 2. Institute of Fluid Mechanics and Aerodynamics, Technische Universität Darmstadt, Darmstadt 64287, Germany
}

Received: February 15, 2016 / Accepted: March 15, 2016 / Published: April 30, 2016.

\begin{abstract}
The characterization of suspension/emulsion sprays plays a decisive role in many industrial processes. A good example of such a process is the drying of a milk spray to produce milk powder, where the process efficiency and product quality is typically controlled by atomization parameters like flow rate, pressure, etc.. However, these parameters influence directly the droplet size and droplet velocity distributions in a spray, so that optimizing a spray drying process often involves adjusting the spray to a desired droplet size and droplet velocity distribution. This requires a measurement technique capable of characterizing in real time, the droplets in a suspension/emulsion spray. To achieve this aim, we present developments to the well-known time-shift technique for spray measurements.
\end{abstract}

Key words: Milk spray, suspension, emulsion, measurement, drop size, drop velocity, time-shift.

\section{Introduction}

The characterization of spray nozzles is one of the important steps in the development of many industrial processes like spray drying, car painting, pharmaceutical coating, etc.. One way to characterize the spray nozzle is to analyze systematically the particle/droplet size and velocity distributions in such sprays. There are numerous methods and techniques which are capable of doing that, a good summary can be found in Tropea (2001) Ref. [1]. In the present study, the time-shift technique has been chosen for spray characterization, because it is capable of measuring not only transparent, but also suspension/emulsion particles/droplets, which is the focus of the present study.

The time-shift technique is a known measurement technique which was first introduced by Semidetnov (1985) [2] and was further developed by Damaschke, et al. (2002) [3, 4]. The time-shift technique has also

Corresponding author: Walter Schäfer, Dr., research field: optical measurement systems for spray characterization. been called the pulse displacement technique, and several variations have been discussed by Hess and Wood (1994) [5], and Lin, et al. (2000) [6]. The new developments and validation of this technique can be found in Refs. [7-9]. A detailed description of the time-shift technique as a measurement method for characterization of suspension/emulsion particles/droplets is discussed extensively in Ref. [10].

The measurement method-time-shift is based on the light scattering of a single droplet/particle from a shaped light beam and can be operated in backscatter, meaning that, the detectors and light sources can be placed on one side of the measurement position, making the technique more attractive for applications with limited optical access. Using the modified time-shift technique $[7,10]$, the milk spray created by different nozzles operated at different pressures will be characterized using the droplet size and droplet velocity distributions of this spray. Milk is an emulsion which contains water and fat particles. So in addition, we will investigate, how the fat content in milk, and with it the viscosity and surface tension, 
influences the droplet size and droplet velocity distribution, depending for instance on nozzle type and pressure. Moreover the time-shift technique is a counting measurement technique like phase Doppler [2], consequently the correlation between velocity and size of the droplets is available.

Using the time-shift technique, several spray nozzles have been analyzed with respect to the measurement of the droplet size and velocity distribution at various liquid feed pressures. These nozzles are typical for spraying suspension/emulsion fluids, for instance milk. Also in this study, milk was used as a working fluid, in order to replicate realistic conditions.

The primary aim of this work is to investigate how the concentration of the dispersed phase in an emulsion influences the atomization process with respect to the particle/droplet size, velocity distributions, spray density and volume flux. A milk water mixture consists mainly of water and fat particles and by diluting with water, the volume density of fat particles can be varied. In addition, this study investigates the influence of operation pressure on the droplet size and velocity distributions as a function of fat concentration in the mixture.

\section{Measurement Principle of the Time-Shift Technique}

The measurement principle of the time-shift technique is only briefly summarized here, since adequate descriptions can be found elsewhere, e.g., Albrecht, et al. (2003) [4]. The measurement principle of the time-shift technique is based on the light scattering of a single particle from a shaped light beam, whereby the size of illuminating beam must be of the same order in size or smaller than the particles to be measured. The light scattered from a single particle can be interpreted as the sum of all scattering orders present at the detector location. With the geometrical optics approximation, this corresponds to examining the propagation of individual rays of light through the particle with varying number of internal reflections.

A typical experimental setup consists of one light source and two photo detectors placed around the light source (Fig. 1). The light is focused by an optical system to a light beam with a small beam waist. The measurement volume is defined by the size of illuminating beam and the imaging optics in front of the detectors. An example signal of a single milk particle passing through the measurement volume is shown in Fig. 2.

In the case of non-transparent particles, e.g., droplets of emulsions, dispersions or suspensions, refracted light rays will no longer propagate undisturbed through the droplet due to absorption or secondary scattering from the dispersed phase, and these rays no longer

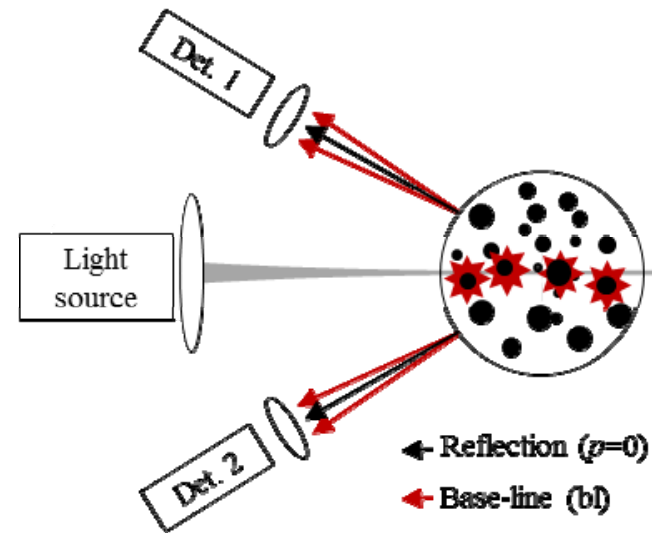

Fig. 1 Schematic representation of the optical configuration.

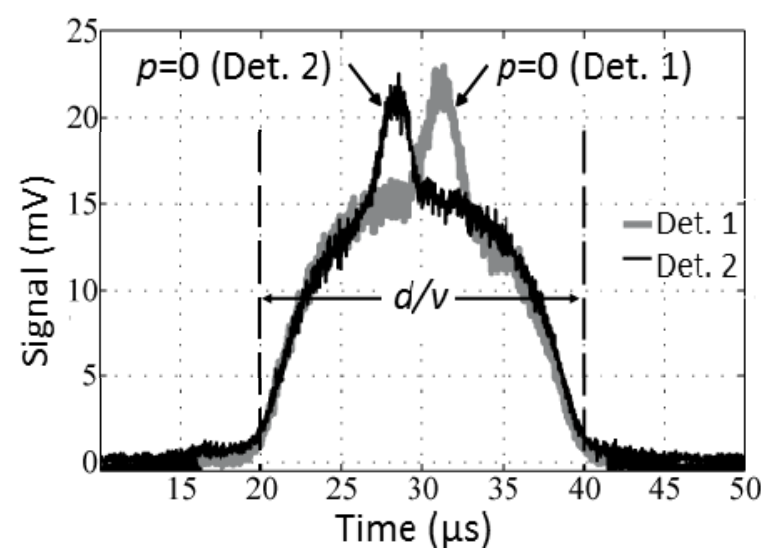

Fig. 2 Time-shift signals obtained from a two detector, time-shift measurement system obtained for a milk droplet passing through the measurement volume. 
contribute directly to the scattered light at a given detector position. Nevertheless, light entering such particles can scatter from scattering centers of the dispersed phase within the particle, and this may result in some scattered light intensity observed at the detector (Fig. 2). The analog signal arising from this portion of scattered light will be termed the baseline signal [10], or signal pedestal.

\section{Experimental Setup}

The optical system is schematically illustrated in Fig. 3. The position of the measurement system is fixed with respect to the spray nozzle, so the measurements of the droplet size and droplet velocity in the spray take place at the same point for all nozzles. In this work, two different spray nozzles were investigated: Schlick $121 \quad 1.7 / 30^{\circ}$ and Schlick 121 $1.7 / 90^{\circ}[11]$. These nozzles are hollow cone spray nozzles with different cone angles: $30^{\circ}$ and $90^{\circ}$. The time-shift setup (SpraySpy® [12]) is mounted to measure the vertical velocity component, hence the nozzles are tilted such that the outer region of the hollow cone spray is directed vertically downwards. The tilt angle of the nozzle is just the half-angle of the spray cone, as shown in Figs. 3 and 4. The measurement point is in the outer portion of the spray. The vertical distance between nozzle and the measurement point is $100 \mathrm{~mm}$. The nozzles have been operated at 2 bar, 3 bar and 4 bar liquid pressure and with water milk mixture with different mixings ratios. For the water milk mixture, the homogenized milk with the fat content of $3.5 \%$ has been used. The measurement matrix is shown in Table 1.

In order to guarantee the comparability of the measurement results, the experimental parameters of the measurement setup like laser power, sensitivity factor, etc., have been fixed and, in addition, the measurement time interval has been kept the same for all measurements. While performing the measurements, it became obvious that, the spray cone changed position slightly with different operating parameters, hence

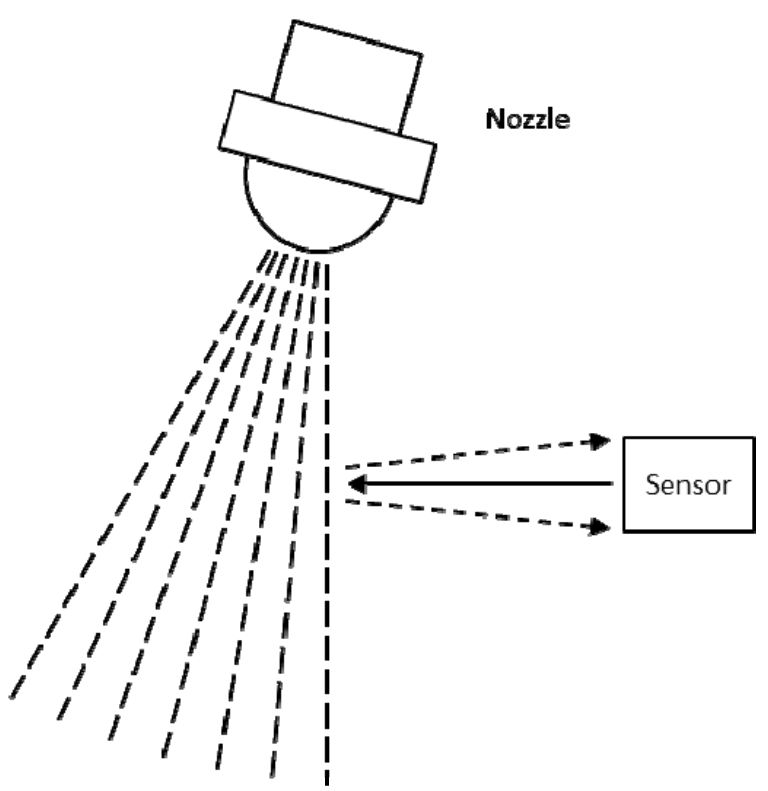

Fig. 3 Schematic illustration of the experimental setup. The spray nozzle is tilted at the half angle of the spray cone angle. Sensor refers to the time-shift setup.

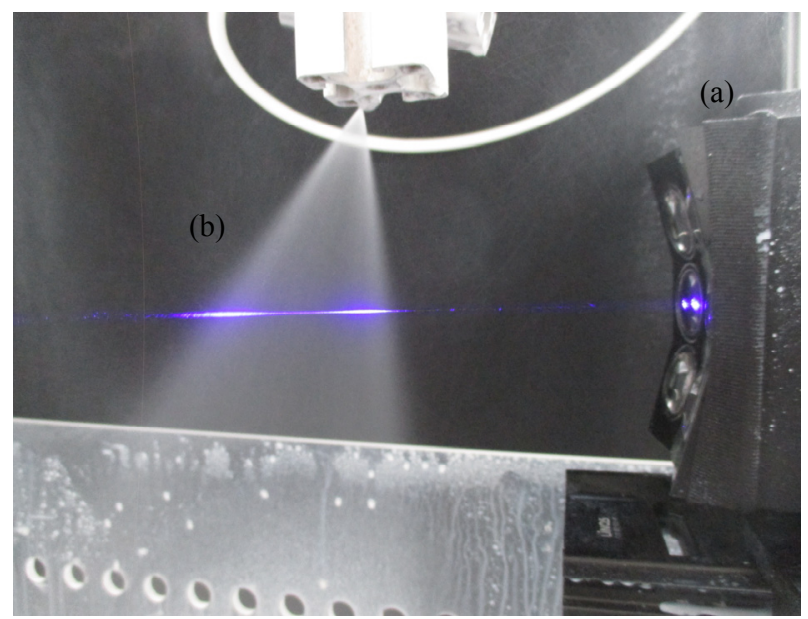

Fig. 4 Picture of the experimental setup: (a) a part of the measurement system (SpraySpy $\left.{ }^{\circledR}\right)$, (b) spray cone from nozzle (Schlick $1211.7 / 30^{\circ}$ ). The nozzle is tilted at $15^{\circ}$.

the measurement position, here fixed in space, exhibited a slight variation relative to the spray cone. This problem was only discovered afterwards and in hindsight, therefore it is recommended to perform traverses prior to the final measurement of each spray to characterize the spatial distribution of the spray cone. This is a problem common to all point measurements techniques and in the meantime standards exist which prescribe a suitable procedure to account for such effects [13]. 
Table 1 Measurement matrix.

\begin{tabular}{|c|c|c|c|c|c|c|c|c|c|c|}
\hline \multirow{2}{*}{$\begin{array}{l}\text { Pressure } \\
\text { (bar) }\end{array}$} & \multirow{2}{*}{$\begin{array}{l}\text { Water } \\
(\%)\end{array}$} & \multirow{2}{*}{$\begin{array}{l}\text { Milk } \\
(\%)\end{array}$} & \multicolumn{4}{|c|}{ Schlick 121 1.7/30 } & \multicolumn{4}{|c|}{ Schlick 121 1.7/90 } \\
\hline & & & $N r$. & $\begin{array}{l}d_{m} \\
(\mu \mathrm{m})\end{array}$ & $\begin{array}{l}v_{m} \\
(\mathrm{~m} / \mathrm{s})\end{array}$ & $\begin{array}{l}\text { Not } \\
\text { valied }\end{array}$ & $N r$. & $\begin{array}{l}d_{m} \\
(\mu \mathrm{m})\end{array}$ & $\begin{array}{l}v_{m} \\
(\mathrm{~m} / \mathrm{s})\end{array}$ & $\begin{array}{l}\text { Not } \\
\text { valied }\end{array}$ \\
\hline 2 & 75 & 25 & 1 & 211 & 14.2 & & 13 & 219 & 12.0 & $x$ \\
\hline 3 & 75 & 25 & 2 & 192 & 17.2 & & 14 & 208 & 14.5 & \\
\hline 4 & 75 & 25 & 3 & 188 & 20.5 & & 15 & 186 & 16.1 & \\
\hline 2 & 50 & 50 & 4 & 219 & 15.1 & & 16 & 233 & 12.0 & \\
\hline 3 & 50 & 50 & 5 & 190 & 17.6 & & 17 & 201 & 14.1 & \\
\hline 4 & 50 & 50 & 6 & 185 & 19.5 & & 18 & 202 & 16.8 & \\
\hline 2 & 25 & 75 & 7 & 244 & 15.6 & $x$ & 19 & 216 & 11.6 & $x$ \\
\hline 3 & 25 & 75 & 8 & 213 & 18.6 & & 20 & 200 & 14.3 & $x$ \\
\hline 4 & 25 & 75 & 9 & 188 & 20.7 & & 21 & 181 & 15.8 & $x$ \\
\hline 2 & 0 & 100 & 10 & 248 & 15.4 & & 22 & 217 & 11.7 & \\
\hline 3 & 0 & 100 & 11 & 221 & 18.4 & & 23 & 201 & 13.8 & \\
\hline 4 & 0 & 100 & 12 & 178 & 20.3 & & 24 & 168 & 14.2 & $x$ \\
\hline
\end{tabular}

$N r$. - the internal serial number of the measurements, $d_{m}$-median of droplet size distribution, $v_{m}$-median of droplet velocity distribution.

\section{Measurement Data Analysis}

The time-shift technique is a counting technique, consequently it measures single droplets which pass successively through the measurement volume, so the information about the size, velocity and acquisition time (the so called time stamp) is assigned to each droplet.

Accordingly, this information can be used to compute the correlation between size and velocity. Furthermore, using the time stamp, the time interval between two measured particles/droplets can be calculated, which offers the possibility to estimate the spray number density or the volume flux. The number of detected particles/droplets per time is a good indicator of the spray number density if the size of the measurement volume is known.

The relative density of particles/droplets can be estimated by:

$$
\Omega \propto \frac{N}{T}
$$

and the relative mass flow can be written as:

$$
\Phi \propto \frac{\sum_{i=0}^{N} d_{i}^{3}}{T}
$$

where, $N$ is the number of detected particles/droplets,
$T$ is the corresponding measurement time, $d_{i}$ is the diameter of the measured particles/droplets. These expressions are only proportionalities and can be interpreted as relative estimates of number and mass flux densities. For absolute estimates of these quantities, the measurement volume size must be known precisely. The measurement time for each point is approximately $20 \mathrm{~s}$. In this time, more than 1,000 droplets have been detected. From the size and velocity distributions, additional parameters can be extracted, such as median droplet size $d_{m}$ and median droplet velocity $v_{m}$ (Table 1). The droplet size distribution is described often by the log-normal function and the droplet velocity distribution by the Gaussian function. Figs. 5 and 6 show these measured distributions with fitted log-normal and Gaussian functions.

The statistical uncertainty of the median measurement values can be estimated using the variance of the individual samples, however in this case, the running exhibits variations above a certain prescribed value after the measurement duration ( $20 \mathrm{~s})$, the measurement is considered valid. Otherwise the measurement is discarded, these measurements are marked "not valid" in Table 1. These measurements are no longer considered in the subsequent data analysis described below. 


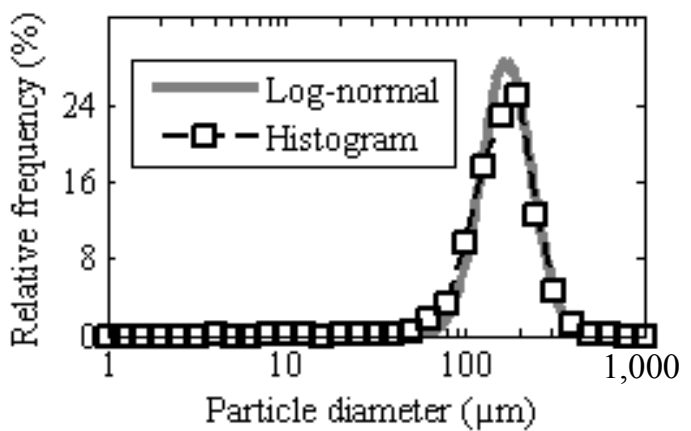

Fig. 5 Droplet size distribution with fitted log normal function nozzle.

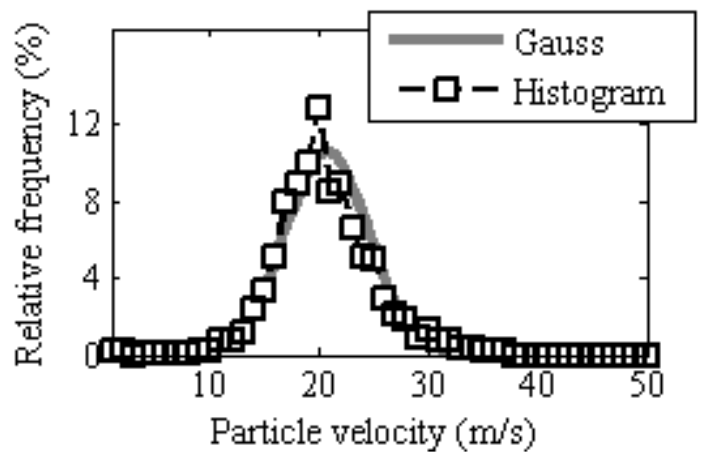

Fig. 6 Droplet velocity distribution with fitted Gaussian function.

\section{Measurement Results}

The correlations between liquid pressure and median values of droplet size and velocity distributions at constant milk concentration are shown in Figs. 7a and $7 \mathrm{~b}$. In addition, the connection between droplet density and volume flux to the milk concentration at constant pressure are shown in Figs. $7 \mathrm{c}$ and $7 \mathrm{~d}$.

The first results depicted in Figs. $7 \mathrm{a}$ and $7 \mathrm{~b}$ show the median droplet velocity and median droplet size as a function of liquid pressure for Schlick $1211.7 / 30^{\circ}$ and Schlick 121 1.7/90 nozzles and for different milk concentration. Independent of milk concentration and nozzle type an increase pressure leads generally to higher velocities of the droplets and to smaller sizes, which is expected from sprays injected into ambient air. The droplet velocity stays approximately constant for different milk-water mixtures, whereas the droplet size is a function of the water content in the milk, especially for the low liquid pressure, as can be shown in Fig. 7a. Additionally in Fig. 7b, it is shown that, for all milk concentrations, the spray nozzle with $90^{\circ}$ spray cone angle has a lower median droplet velocity than the nozzle with a cone angle of $30^{\circ}$. This might be caused by different flow rates of nozzles at the same liquid pressure. The nozzle Schlick 121 1.7/90 exhibits a higher flow rate at the same pressure as the nozzle Schlick 121 1.7/30.

In addition, the surface of the spray cone of the nozzle with $30^{\circ}$ is smaller than of the nozzle with $90^{\circ}$ spray cone. This infers that, the liquid volume with the nozzle Schlick $121 \quad 1.7 / 90^{\circ}$ is spatially more distributed, which results in a lower spray number density, volume flux and lower velocity. This effect can be seen on the basis of data depicted in Figs. 7c and 7 d. Fig. $7 \mathrm{c}$ shows the function omega (Eq. (1)) which describes the droplet density in the spray. In Fig. 7c, it can be seen that, the spray density (omega) of the nozzle: Schlick $1211.7 / 30^{\circ}$ is significant larger than by the nozzle: Schlick $1211.7 / 90^{\circ}$. Subsequently the function phi exhibits the same behavior in Fig. 7d.

In the next step, we use the same data to analyze the correlation between key parameters and the milk concentration by different liquid pressure. The results are depicted in Fig. 8. Figs. 8a and $8 \mathrm{~b}$ show how the content of fat particles in the water milk mixture influences the droplet size and velocity. The median velocity $v_{m}$ stays approximately constant over different milk concentrations, which can be observed already in Fig. 7b. On the other hand, $d_{m}$ has to be a function of the milk concentration, which for different nozzles exhibits an opposite dependency, as shown in Fig. 8a. The $d_{m}$ of the nozzle with the spray cone angle of $90^{\circ}$ decreases slightly with increasing milk concentration, and $d_{m}$ of the nozzle with the cone angle of $30^{\circ}$ increases with increasing milk concentration. The differences of omega and phi can be also seen in Figs. 8c and 8d. The nozzle Schlick $1211.7 / 30^{\circ}$ has approximately the same values of phi and omega independent of the milk concentration, whereas the nozzle Schlick $1211.7 / 90^{\circ}$ has a positive dependency at 4 bar liquid pressure. 


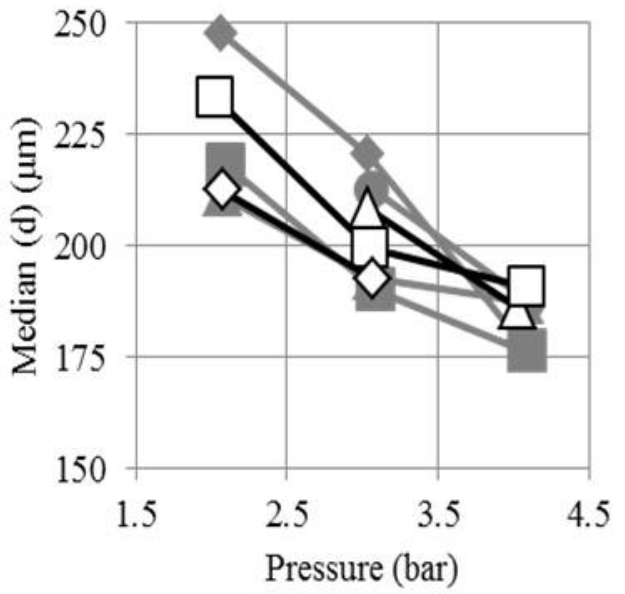

(a)

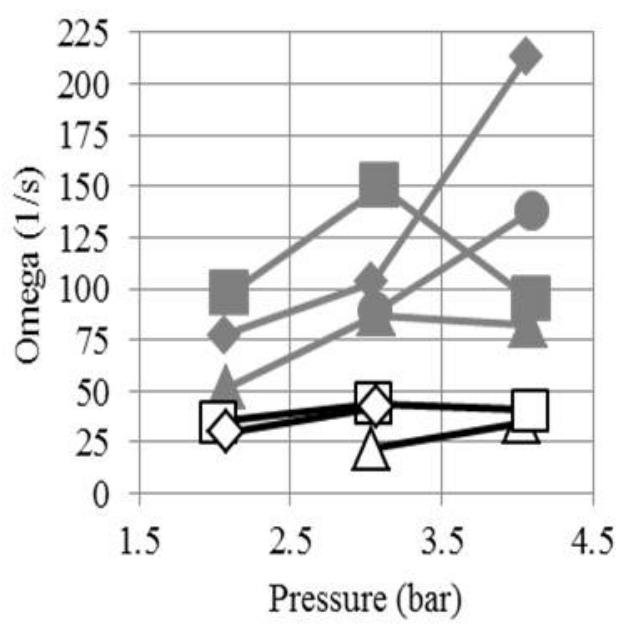

(c)

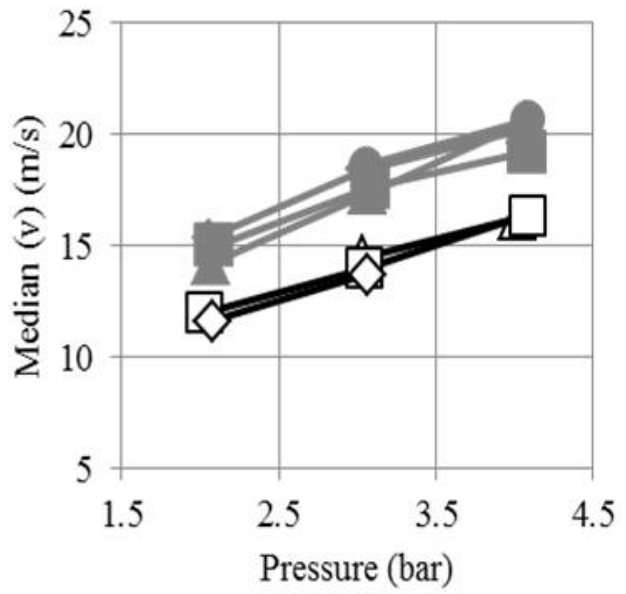

(b)

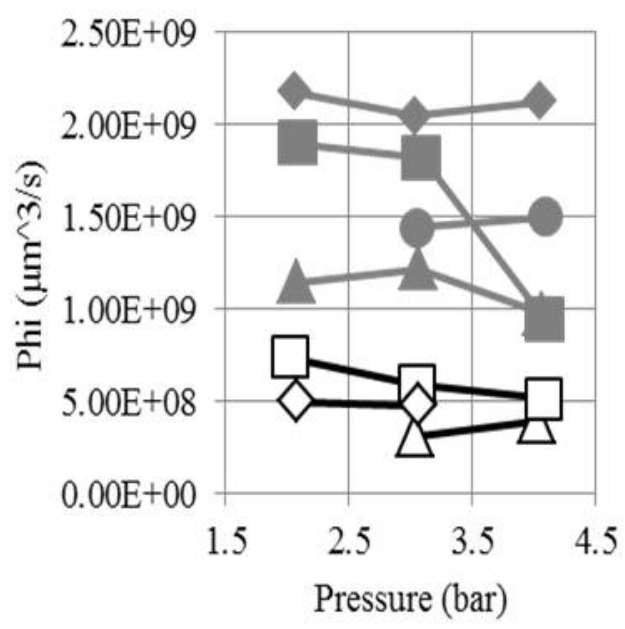

(d)

Fig. 7 Median values of (a) droplet size and (b) velocity distributions, (c) the spray density estimator omega (Eq. (1)) and (d) the volume flux phi (Eq. (2)) as a function of liquid pressure for two nozzles and different milk concentration. Nozzle $30^{\circ}$ represents Schlick $1211.7 / 30^{\circ}$ and nozzle $90^{\circ}$ represents Schlick $1211.7 / 90^{\circ}$. 


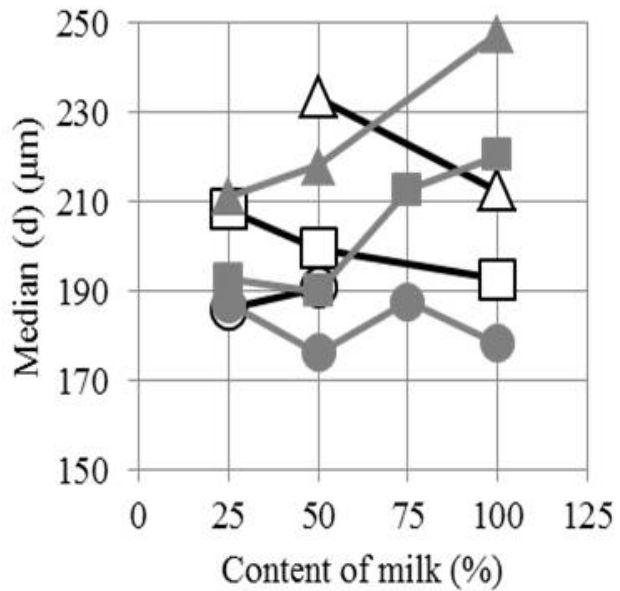

(a)

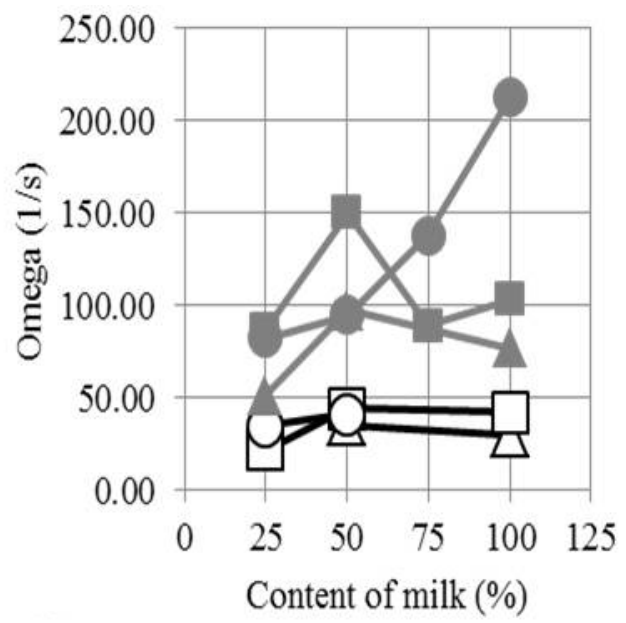

(c)

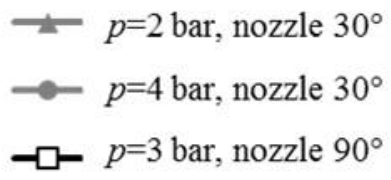

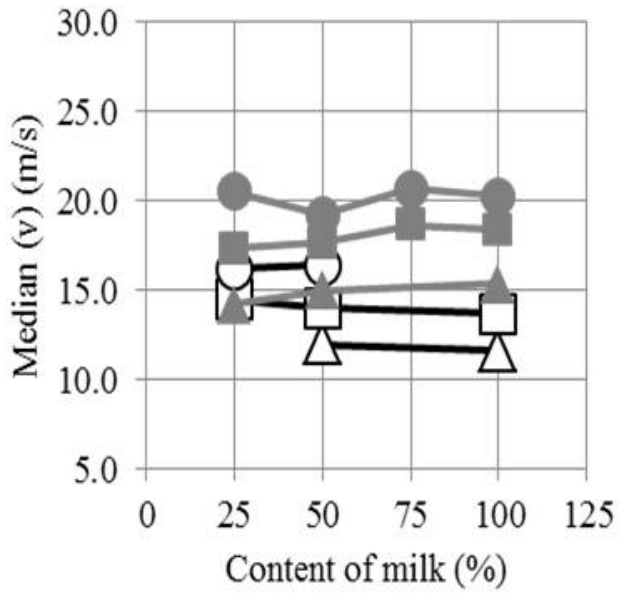

(b)

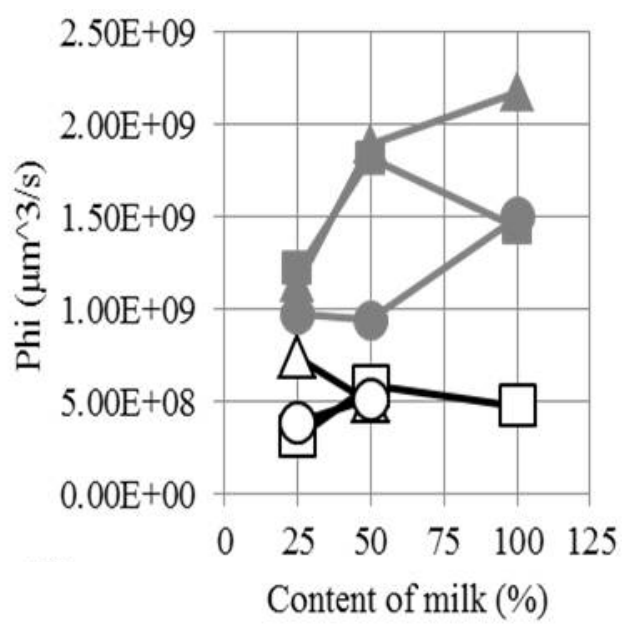

(d)

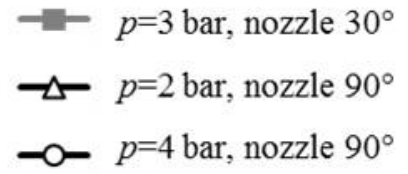

Fig. 8 Median values of (a) droplet size and (b) velocity distributions, (c) the spray density estimator omega (Eq. (1)) and (d) the volume flux phi (Eq. (2)), a function of milk concentration for two nozzles and different liquid pressure. Nozzle $30^{\circ}$ represents Schlick $1211.7 / 30^{\circ}$ and nozzle $90^{\circ}$ represents Schlick 121 1.7/90 ${ }^{\circ}$.

\section{Summary and Conclusions}

In this study, we present a demonstration of the time-shift technique for characterization of droplets in a milk spray. This technique is quite promising and can also be used for estimating of the relative number density and volume flux in the spray. In this work, we characterized two nozzles and present correlations between milk concentration and key parameters quantifying the spray. We found that, the measurement at a single point is a problem, if the spray geometry changes with operating parameters. In such cases, it is recommended to conduct a spatial scan to first characterize the spray and chose an appropriate measurement position.

\section{References}

[1] Tropea, C. 2011. "Optical Particle Characterization in Flows." Annual Review of Fluid Mechanics 43 (January): 399-426. 
[2] Semidetnov, N. 1985. "Investigation of Laser Doppler Anemometer as Instrument for Two-Phase Flow Measurements (in Russia)." Leningrad Institut for Precision Mechanics and Optics.

[3] Damaschke, N., Nobach, H., Semidetnov, N., and Tropea, C. 2002. "Optical Particle Sizing in Backscatter." Appl. Opt. 41 (27): 5713-27.

[4] Albrecht, H. E., Damaschke, N., Borys, M., and Tropea, C. 2002. Laser Doppler and Phase Doppler Measurement Techniques. Berlin Heidelberg: Springer.

[5] Hess, C. F., and Wood, C. P. 1994. The Pulse Displacement Technique-A Single Particle Counter with a Size Range Larger than 1000: 1." Particle \& Particle Systems Characterization 11 (1): 107-13.

[6] Lin, S. M., Waterman, D. R., and Lettington, A. H. 2000. "Measurement of Droplet Velocity, Size and Refractive Index Using the Pulse Displacement Technique." Measurement Science and Technology 11 (6): L1-L4.

[7] Schäfer, W. 2013. "Time-Shift Technique for Particle Characterization in Sprays." Technische Universität Darmstadt.
[8] Schäfer, W., and Tropea, C. 2014. "Time-Shift Technique for simultaneous Measurement of Size, Velocity, and Relative Refractive Index of Transparent Droplets or Particles in a Flow." Applied Optics 53 (4): 588.

[9] Schäfer, W., Rosenkranz, S., and Tropea, C. 2015. "Validation of the Time-Shift Technique for Spray Characterization." Presented at the ILASS Americas 27th Annual Conference on Liquid Atomization and Spray Systems, Raleigh, NC, USA.

[10] Schäfer, W., and Tropea, C. 2014. "The Time-Shift Technique for Measurement Size of Non-transparent Spherical Particles." In Proceedings of the SPIE (the International Society for Optics and Photonics), 92320H.

[11] Düsen-Schlick GmbH, Hutstrasse 4, 96253 Untersiemau/Coburg, Germany.

[12] AOM-Systems GmbH, Flughafenstrasse 15, 64347 Griesheim, Germany.

[13] DIN SPEC (German Institute for Standardization) 91325. 2015. Characterization of Sprays and Spraying Processes by Measuring the Size and Velocity of Non-transparent Droplets. Berlin: Beuth Verlag. 